\title{
Comparison of sugammadex and pyridostigmine bromide for reversal of rocuronium-induced neuromuscular blockade in short-term pediatric surgery: a prospective randomized study
}

Received October 24, 2018

Revised 1st, October 29, 2018 2nd, October 30, 2018 Accepted October 30, 2018

\section{Corresponding author}

Jihyun An, M.D.

Department of Anesthesiology and Pain Medicine, Daegu Fatima Hoapital, 99 Ayang-ro, Dong-gu,

Daegu 41199, Korea

Tel: 82-53-940-7429

Fax: 82-53-940-7443

E-mail: anji43@naver.com

\section{ORCID}

https://orcid.org/0000-0002-5373-3887

\section{Jihyun An, Eunju Kim, Jihyang Lee, Hyun Kim, Jongcheol Son, Joonyoung Huh, and Kyeongyoon Woo}

Department of Anesthesiology and Pain Medicine, Daegu Fatima Hospital, Daegu, Korea
Background: Sugammadex reverses rocuronium-induced neuromuscular blockade quickly and effectively. Herein, we compared the efficacy of sugammadex and pyridostigmine in the reversal of rocuronium-induced light block or minimal block in pediatric patients scheduled for elective entropion surgery.

Methods: A prospective randomized study was conducted in 60 pediatric patients aged 2-11 years who were scheduled for entropion surgery under sevoflurane anesthesia. Neuromuscular blockade was achieved by administration of $0.6 \mathrm{mg} / \mathrm{kg}$ rocuronium and assessed using the train-of-four (TOF) technique. Patients were randomly assigned to 2 groups receiving either sugammadex $2 \mathrm{mg} / \mathrm{kg}$ or pyridostigmine $0.2 \mathrm{mg} / \mathrm{kg}$ and glycopyrrolate $0.01 \mathrm{mg} / \mathrm{kg}$ at the end of surgery. Primary outcomes were time from administration of reversal agents to TOF ratio 0.9 and TOF ratio 1.0. Time from the administration of reversal agents to extubation and postoperative adverse events were also recorded.

Results: There were no significant differences in the demographic variables. Time from the administration of reversal agents to TOF ratio 0.9 and TOF ratio 1.0 were significantly shorter in the sugammadex group than in the pyridostigmine plus glycopyrrolate group: $1.30 \pm 0.84$ vs. $3.53 \pm 2.73 \mathrm{~min}(P<0.001)$ and $2.75 \pm 1.00$ vs. $5.73 \pm 2.83 \min (P$ $<0.001$ ), respectively. Extubation time was shorter in the sugammadex group. Adverse events, such as skin rash, nausea, vomiting, and postoperative residual neuromuscular blockade (airway obstruction), were not statistically different between the two groups.

Conclusions: Sugammadex provided more rapid reversal of rocuronium-induced neuromuscular blockade in pediatric patients undergoing surgery than did pyridostigmine plus glycopyrrolate.

Keywords: Delayed emergence from anesthesia; Neuromuscular monitoring; Pediatrics; Pyridostigmine bromide; Rocuronium; Sugammadex.

\section{INTRODUCTION}

Sugammadex is an alternative to cholinesterase inhibitors used in the reversal of neuromuscular blockade. Postoperative residual neuromuscular blockade and adverse effects related to muscarinic receptors are not observed when sugammadex is used to reverse rocuronium-induced neuromuscular blockade [1]. However, pharmacokinetic and pharmacodynamic properties of neuromuscular blockade in children differ from those in adults due to the larger volume

This is an Open Access article distributed under the terms of the Creative Commons Attribution Non-Commercial License (http://creativecommons.org/licenses/by-nc/4.0) which permits unrestricted non-commercial use, distribution, and reproduction in any medium, provided the original work is properly cited. 
of distribution and the presence of immature neuromuscular receptors [2]. Therefore, studies on the reversal of neuromuscular block in pediatric patients should be conducted separately from those in adults. A study comparing sugammadex with neostigmine was recently published [3-5], but comparisons of sugammadex with pyridostigmine in pediatric anesthesia are rare. A previous study has compared the effect of reversal agents administered at moderate neuromuscular blockade (Intense block: post-tetanic count 0 and train-of-four [TOF] count 0, Deep block: post-tetanic count $\geq 1$ and TOF count 0, Moderate block: TOF count 1-3, Minimal block: TOF ratio 0.1-0.4, Light block: $0.4<$ TOF ratio < 0.9) [6]. However, in short surgery without re-administration of rocuronium, minimal or light neuromuscular blockade is often observed at the end of surgery $[6,7]$.

This study aimed to compare the efficacy of sugammadex and pyridostigmine in reversing light block or minimal block in pediatric patients scheduled for elective entropion surgery.

\section{MATERIALS AND METHODS}

This prospective, randomized study was performed after obtaining approval from the ethics committee of Daegu Fatima Hospital (no. DFH17MRIO335) and obtaining written consent from the person legally responsible for each child. Sixty healthy pediatric patients, as per the American Society of Anesthesiologists physical status classification I/II, aged 2-11 years and scheduled for entropion surgery were enrolled in this study. Exclusion criteria were developmental delays, intellectual disability, anxiety disorder, chronic illness (renal disease, liver disease, neuromuscular disease, etc.), previous anesthetic experience, known malignant hyperthermia in the family history, and upper respiratory infection symptom.

Randomization was performed using a random number table generated by Microsoft Excel (Microsoft Corp., USA). Patients were classified into 2 groups each on the basis of the reversal agent used: sugammadex (Group S) or pyridostigmine plus glycopyrrolate (Group P).

A researcher blinded to the 2 types of reversal agents in syringes containing the same amount ( $3 \mathrm{ml}$ ) injected the reversal agents. An anesthesiologist responsible for TOF monitoring and data collection was blinded to the administration of the neuromuscular blockade reversal agents.

Both groups were administered intravenous atropine as premedication $30 \mathrm{~min}$ before surgery. In the operating room, basic monitoring was carried out as follows: electrocardiogram, noninvasive blood pressure, pulse oximetry, and capnography. Subsequently, ketamine $2 \mathrm{mg} / \mathrm{kg}$ was administered for induction of general anesthesia. Neuromuscular monitoring was applied after injection of ketamine to avoid pain in pediatric patients.

Neuromuscular block was monitored with the TOF Watch SX (Organon, Ireland) using the acceleromyographic response of the adductor pollicis muscle to repetitive TOF stimulation of the ulnar nerve using surface electrodes. The peripheral heat sensor was placed on the ventral side of the hand. The acceleromyographic transducer was positioned on the palmar side on the top of the thumb. The monitored arm was immobilized on the operating table. Stabilization was achieved within $5 \mathrm{~s}$ at $50-\mathrm{Hz}$ tetanic stimulation; 1 min after tetanic stimulation, the fingers-excluding the thumbwere fixated and completely immobilized. After 1 min of TOF stimulation, calibration was achieved by pressing the CAL 2 button. After calibration, supramaximal stimulation was applied (current $<60 \mathrm{~mA}$ ) with square wave pulses of $0.2 \mathrm{~ms}$ duration that were delivered at $2 \mathrm{~Hz}$ at intervals of $15 \mathrm{~s}$ during surgery; at the end of surgery the TOF monitor was removed after confirmation of TOF ratio $=1.0$.

After the application of neuromuscular monitoring, rocuronium $0.6 \mathrm{mg} / \mathrm{kg}$ was administered. Fentanyl $1 \mu \mathrm{g} / \mathrm{kg}$ was used for endotracheal intubation in both groups, and dexamethasone $0.2 \mathrm{mg} / \mathrm{kg}$ was administered to prevent postoperative sore throat and spasm. Intubation was performed by the anesthesiologist after confirmation of TOF count 0 .

Anesthesia was maintained with a fraction of inspired oxygen 0.5 and $2-3 \%$ sevoflurane. If the surgery was expected to last longer than $30 \mathrm{~min}$, another bolus dose of rocuronium 0.2 $\mathrm{mg} / \mathrm{kg}$ was administered after the first $40 \mathrm{~min}$. The total dose of rocuronium and the time of the last dose of rocuronium were recorded.

At the end of the surgery, sugammadex $2 \mathrm{mg} / \mathrm{kg}$ or the combination of pyridostigmine $0.2 \mathrm{mg} / \mathrm{kg}$ with glycopyrrolate $0.01 \mathrm{mg} / \mathrm{kg}$ was injected after confirming TOF ratio $\geq 0.1$ (minimal to light block) and sevoflurane was discontinued. If neuromuscular blockade degree at the end of surgery was TOF count 0-3 (intense to moderate block), sevoflurane was continued with 0.4 minimum alveolar concentration until reappearance of fourth twitch (T4). If the TOF ratio did not 
reach 0.9 after $10 \mathrm{~min}$ of reversal agent administration or if postoperative neuromuscular blockade symptoms were present, the rescue dose which is equal to the initial dose was administered. Time of administration of sugammadex or pyridostigmine-glycopyrrolate after last rocuronium administration and TOF ratio at that time were recorded. Tracheal extubation was performed when TOF ratio $>1.0$ and spontaneous ventilation (tidal volume $>5 \mathrm{ml} / \mathrm{kg}$ ) was maintained with appropriate oxygenation and ventilation as measured by breathing capnography, hemodynamic stability, and recovery of response to verbal commands. Extubation time was recorded. After extubation, each patient was transferred to the post-anesthesia care unit (PACU). Propacetamol $30 \mathrm{mg} /$ $\mathrm{kg}$ mixed with $50 \mathrm{ml}$ normal saline was administered for 20 min for postoperative pain control. If the visual analog scale score was $\geq 6$ or the patient was agitated or physical restraint was required, fentanyl $1 \mu \mathrm{g} / \mathrm{kg}$ was administered.

Primary outcomes were time from administration of reversal agents to TOF ratio 0.9 and TOF ratio 1.0. Secondary outcomes were time from administration of reversal agent to extubation, TOF ratio 0.9 to extubation, time from TOF ratio 1.0 to extubation, administration of additional reversal agents, and adverse events. Adverse events included bradycardia ( $>20 \%$ decrease from baseline heart rate), tachycardia (increased $>20 \%$ from baseline heart rate), hypotension (mean blood pressure $<50 \mathrm{mmHg}$ ), nausea, vomiting, diplopia, fever, skin rash, dry mouth, and postoperative residual neuromuscular blockade in the PACU. Postoperative neuromuscular blockade adverse events included upper airway obstruction requiring intervention, hypoxemia ( $<94 \%$ oxygen saturation), and reintubation.

\section{Statistical analysis}

Power and sample calculators (http://www.powerandsamplesize.com/Calculators/) were used for sample size calculation. In a study by Kara et al. [4], recovery time (time to TOF ratio 0.9 ) was $0.46 \pm 0.70 \mathrm{~min}$ for the sugammadex group versus $1.97 \pm 2.14 \mathrm{~min}$ for the neostigmine group. The suggested minimum sample size was 20 per sugammadex group and 20 per pyridostigmine group at power $=0.9, \alpha=0.05$. Considering $35 \%$ of dropouts and failures, 32 subjects were recruited in each group.

Student's $t$-tests were used to compare variables that were normally distributed such as height, weight, age (month), surgery time, anesthesia time, a dose of rocuronium, the interval from last rocuronium to reversal agent, TOF ratio, and time variations. Mann-Whitney $U$ tests were used to compare variables that were not normally distributed such as temperature on palm and mid-esophagus. Fisher's exact chi-squared tests were used to examine the development of adverse events in both groups. All statistical analyses were performed with IBM SPSS statistics version 21.0 (IBM, USA). Statistical significance was set at $\mathrm{P}<0.05$.

\section{RESULTS}

Sixty-four subjects were recruited in this study from 1 September 2017 to 30 June 2018; 4 patients were excluded after refusal by patients' guardians. Patients were classified into 2 groups of 30 patients each on the basis of the reversal agent used: sugammadex (Group S) or pyridostigmine plus glycopyrrolate (Group P). There were no significant differences in demographic variables between the groups (Table 1). The palm temperatures before injection of reversal agents were not statistically different between the groups (Group S: $35.7^{\circ} \mathrm{C}$ [35.4, 35.9 ${ }^{\circ} \mathrm{C}$; Group P: $35.7^{\circ} \mathrm{C}\left[35.1,35.9^{\circ} \mathrm{C}\right] ; \mathrm{P}=0.826$ ) (Table 2). TOF ratios before administration of reversal agents were not statistically different between the groups (Group S: 23.92 \pm 23.73 ; Group P: $32.50 \pm 22.48, \mathrm{P}=0.152$ ) (Table 3). The mean time from administration of reversal agent to TOF ratio 0.9 was about 2.5 fold shorter for Group S $(\mathrm{P}<0.001)$ than it was for Group P. The mean time from administration of reversal agent to TOF ratio 1.0 was also about 2 fold shorter for Group $\mathrm{S}$ than for Group $\mathrm{P}(\mathrm{P}<0.001)$ (Table 3$)$. The mean time from the administration of reversal agent to extubation was about 2 min shorter for Group S (P = 0.022) (Table 3). Mean anes-

Table 1. Demographic Data organized by Group

\begin{tabular}{lcc}
\hline \multicolumn{1}{c}{ Variable } & Group $S(\mathrm{n}=30)$ & Group $\mathrm{P}(\mathrm{n}=30)$ \\
\hline Sex, M/F & $19(63.3) / 11(36.7)$ & $15(50.0) / 15(50.0)$ \\
Height $(\mathrm{cm})$ & $118.7 \pm 17.0$ & $118.0 \pm 15.7$ \\
Weight $(\mathrm{kg})$ & $25.7 \pm 10.6$ & $27.0 \pm 13.3$ \\
Age $(\mathrm{mo})$ & $77.9 \pm 26.4$ & $77.6 \pm 27.5$
\end{tabular}

Values are presented as number (\%) or mean \pm SD. Student's $t$-tests were performed, with values presented as mean \pm SD. Chi-squared and Fisher's exact tests were performed for sex, with values presented as number (\%). Group S: sugammadex group, Group P: pyridostigmine group. 
Table 2. Intraoperative Parameters

\begin{tabular}{lccc}
\hline \multicolumn{1}{c}{ Variable } & Group S $(\mathrm{n}=30)$ & Group P $(\mathrm{n}=30)$ & $\mathrm{P}$ value \\
\hline Surgery time $(\mathrm{min})$ & $52.48 \pm 25.67$ & $50.65 \pm 21.09$ & 0.490 \\
Anesthesia time $(\mathrm{min})$ & $76.42 \pm 28.12$ & $81.10 \pm 26.61$ & 0.819 \\
Dose of rocuronium $(\mathrm{mg})$ & $16.5 \pm 6.79$ & $16.08 \pm 7.13$ & 0.818 \\
Additional rocuronium administration & $8(26.7)$ & $6(20)$ & 0.761 \\
Interval from last rocuronium to reversal agent (min) & $57.80 \pm 17.93$ & $62.87 \pm 16.18$ & 0.255 \\
Temperature on palm $\left({ }^{\circ} \mathrm{C}\right)$ & $35.7(35.4,35.9)$ & $35.7(35.1,35.9)$ & 0.826 \\
Temperature on mid-esophagus $\left({ }^{\circ} \mathrm{C}\right)$ & $36.9(36.7,37.0)$ & $36.9(36.7,37.0)$ & 0.407 \\
\hline
\end{tabular}

Values are presented as mean \pm SD, number (\%), or median (1Q, 3Q). Student's $t$-tests were performed, with values presented as mean \pm SD. Mann-Whitney $U$ test was performed for temperatures on palm and mid-esophagus and are presented as median (1Q, 3Q). Chi-squared and Fisher's exact tests were performed for additional rocuronium administration, with values presented as number (\%). Group S: sugammadex group, Group P: pyridostigmine group.

Table 3. Train-of-Four (TOF) Ratio and Evaluation of Time Variations, by Group

\begin{tabular}{lccc}
\hline \multicolumn{1}{c}{ Variable } & Group S $(\mathrm{n}=30)$ & Group P $(\mathrm{n}=30)$ & $\mathrm{P}$ value \\
\hline Initial TOF (\%) & $102.2 \pm 7.37$ & $99.8 \pm 6.95$ & 0.212 \\
TOF at the administration of reversal agent $(\%)$ & $23.92 \pm 23.73$ & $32.50 \pm 22.48$ & 0.152 \\
Time from reversal agent to TOF ratio 0.9 $(\mathrm{min})$ & $1.30 \pm 0.84$ & $3.53 \pm 2.73$ & $<0.001$ \\
Time from reversal agent to TOF ratio 1.0 (min) & $2.75 \pm 1.00$ & $5.73 \pm 2.83$ & $<.001$ \\
Time from TOF ratio 0.9 to extubation (min) & $4.94 \pm 3.18$ & $5.11 \pm 3.78$ & 0.864 \\
Time from TOF ratio 1.0 to extubation (min) & $4.10 \pm 2.75$ & $3.26 \pm 2.23$ & 0.202 \\
Time from reversal agent to extubation $(\mathrm{min})$ & $6.23 \pm 3.48$ & $8.58 \pm 3.89$ & 0.022 \\
\hline
\end{tabular}

Values are presented as mean \pm SD. Statistical analysis was performed using the Student's $t$-test. Group S: sugammadex group, Group P: pyridostigmine group.

thesia time was 4.68 min shorter for Group S than for Group P but this difference was not statistically significant.

There was no significant difference in the postoperative residual neuromuscular blockade. Upper airway obstructions after extubation such as bronchospasm and laryngospasm occurred in 3 patients in Group S and in 7 patients in Group P. The conditions of 3 patients in Group P did not resolve with positive mask ventilation and improved only after administration of lidocaine $1 \mathrm{mg} / \mathrm{kg}$. However, there was no significant difference in the incidence of upper airway obstruction between the two groups. There was no additional reversal agent and no re-intubation in both groups (Table 4). Two cases of nausea were reported in Group P, but none were reported in Group S; vomiting occurred in 1 patient per group. Three patients in Group P complained of dry mouth, but there were no such reports in Group S (Table 4).

\section{DISCUSSION}

For decades, cholinesterase inhibitors (neostigmine, pyridostigmine, and edrophonium) were used as reversal agents
Table 4. Incidence of Adverse Events Organized by Group

\begin{tabular}{lllc}
\hline \multicolumn{1}{c}{ Variable } & $\begin{array}{l}\text { Group S } \\
(\mathrm{n}=30)\end{array}$ & $\begin{array}{l}\text { Group P } \\
(\mathrm{n}=30)\end{array}$ & P value \\
\hline Nausea & $0(0.0)$ & $2(6.7)$ & 0.492 \\
Vomiting & $1(3.3)$ & $1(3.3)$ & 1.000 \\
Upper airway obstruction & $3(10.0)$ & $7(23.3)$ & 0.299 \\
Skin rash & $1(3.3)$ & $1(3.3)$ & 1.000 \\
Bradycardia & $0(0.0)$ & $0(0.0)$ & N/A \\
Tachycardia & $0(0.0)$ & $0(0.0)$ & N/A \\
Hypotension & $0(0.0)$ & $0(0.0)$ & N/A \\
Dry mouth & $0(0.0)$ & $3(10.0)$ & 0.078 \\
Fever & $0(0.0)$ & $0(0.0)$ & N/A \\
Diplopia & $0(0.0)$ & $0(0.0)$ & N/A \\
Additional administration of & $0(0.0)$ & $0(0.0)$ & N/A \\
$\quad$ reversal agent & & & \\
Reintubation & $0(0.0)$ & $0(0.0)$ & N/A \\
Hypoxemia (<94\%) & $2(6.7)$ & $5(16.7)$ & 0.243 \\
\hline
\end{tabular}

Values are presented as number (\%). Statistical analyses were performed using the chi-squared and Fisher's exact tests. Group S: sugammadex group, Group P: pyridostigmine group.

despite their undesirable effects on the muscarinic receptors. In a recent study, a new reversal agent, sugammadex, was reported which caused no muscarinic effects and had a faster 
recovery time than neostigmine in adults [8]. A meta-analysis by Guangyu et al. [3] reported that compared to neostigmine or a placebo, sugammadex may reverse rocuronium-induced neuromuscular blockade more rapidly with comparable incidence of adverse events in pediatric patients. However, the included studies used sugammadex to reverse moderate or deep neuromuscular blockade and was not available for the shallow block (TOF ratio 0.25 or 0.5 ). In fact, at the end of the surgery, it is common to observe minimal to light neuromuscular blockade as well as a moderate neuromuscular blockade. Therefore, we compared sugammadex with the pyridostigmine used in our institution in minimal to light neuromuscular blockade. The onset of action for pyridostigmine is $16 \mathrm{~min}$, which is longer than the $7-11 \mathrm{~min}$ for neostigmine [9].

We expected that the difference in time from administration of reversal agent to TOF ratio 0.9 between the sugammadex group and pyridostigmine group would be greater than that seen in previous studies comparing neostigmine and sugammadex (in which the recovery time was up to 5 times faster for sugammadex [5]) and that the frequency of postoperative residual neuromuscular blockade would be higher [9] because of the delayed onset of pyridostigmine. The onset of action for pyridostigmine is $16 \mathrm{~min}$, which is longer than the 7-11 min for neostigmine [10]. Unexpectedly, our results showed that the time from administration of reversal agent to TOF ratio 0.9 and TOF ratio 1.0 were only 2 fold faster in case of sugammadex than pyridostigmine and neither group exhibited postoperative residual neuromuscular blockade. In previous studies, additional neuromuscular blockers were administered to maintain a TOF count $<2$, and a reversal agent was administered when the second twitch (T2) of TOF was observed at the end of the procedure $[5,8]$. In our study, additional rocuronium was not administered unless the surgery was expected to continue $>30 \mathrm{~min}$. Additional rocuronium was administered after the first $40 \mathrm{~min}$. As a result, most patients, except three, had already reached a light block or minimal block state at the end of surgery (with the exception of 2 patients in pyridostigmine group who were at T2 and 1 patient in sugammadex group who was at $\mathrm{T} 0$; these 3 patients received a reversal agent when T4 of TOF appeared). This is the reason there was less difference in the time from administration of reversal agent to TOF ratio 0.9 between the 2 groups than was observed in the previous study. As in other studies which compared sugammadex and neostigmine in moderate block $[5,8,11]$, use of sugammadex in minimal to light block state induced effective reversal and reduced the time to TOF ratio 0.9.

Kara et al. [4] reported that the time from reversal agent to extubation was 2 fold shorter for the sugammadex group. In another study, extubation time was $202.5(45,515) \mathrm{s}$ in the sugammadex group and $427(17.5,720) \mathrm{s}$ in the neostigmine group [12]. In our study, time from reversal agent to extubation was 2 min shorter in the sugammadex group $(6.23 \pm 3.48$ min) compared with pyridostigmine group ( $8.58 \pm 3.89, \mathrm{P}=$ 0.022). Although the time to extubation was shorter in the sugammadex group as in previous studies, the difference was smaller. This is a result of the shallow neuromuscular blockade state compared to the previous study. In addition, the synergistic effect of fentanyl and sevoflurane used at induction resulted in the extension of extubation time compared to a previous study [13]. Further, the difference in extubation criteria may affect extubation time. The mid-esophageal temperature measured at the end of surgery was normal in both groups, so it did not affect time from TOF 0.9 to extubation and time from TOF 1.0 to extubation.

This study has certain limitations. First, sugammadex is a drug that has not yet been approved by the Korean Food and Drug Administration in pediatric patients under 18 years of age. Therefore, the study started after the approval of the Korean Food and Drug Administration for the research. Further studies are needed to demonstrate the safety of the use of sugammadex in pediatric patients. Second, the sample size was obtained from a study comparing sugammadex with neostigmine. Third, there was difficulty in finding statistical differences between the 2 groups in terms of adverse effects because the number of patients participating in the study was small. Further, dexamethasone administration at induction may affect the frequency of nausea, vomiting, and upper airway obstruction. Fourth, the sevoflurane concentration data was not collected. Sevoflurane may enhance the effect of rocuronium and affect the reversal of rocuronium-induced neuromuscular blockade [14]. In addition, it was difficult to measure adverse events because the subjects were pediatric patients who were not as efficient as adults in reporting symptoms. Even when patients felt dizzy, nauseous, or in pain, they could not properly express their symptoms, and they exhibited nonspecific symptoms such as restlessness, 
grimacing, struggling, and crying. The emergence of agitation may be the consequence of other etiologies, including hypoxemia, pain, bladder distension, and nausea [15]. Therefore, it would be necessary to include the evaluation of postoperative agitation on the pediatric anesthesia emergence delirium scale in further studies.

Reversal of rocuronium-induced neuromuscular blockade and extubation time were more rapid with sugammadex than with pyridostigmine plus glycopyrrolate in pediatric patients undergoing short surgery with surgery time of around 40-60 min. Overall differences in anesthesia time were statistically insignificant. There were no statistical differences in frequency of adverse events between reversal with sugammadex and reversal with pyridostigmine plus glycopyrrolate. In shortterm pediatric surgery, sugammadex can be used instead of pyridostigmine and can be useful when needed for fast reversal.

\section{CONFLICTS OF INTEREST}

No potential conflict of interest relevant to this article was reported.

\section{ORCID}

Eunju Kim: https://orcid.org/0000-0002-7299-4644

Jihyang Lee: https://orcid.org/0000-0001-5038-8419

Hyun Kim: https://orcid.org/0000-0003-4994-1568

Jongcheol Son: https://orcid.org/0000-0001-8884-7422

Joonyoung Huh: https://orcid.org/0000-0001-7106-2303

Kyeongyoon Woo: https://orcid.org/0000-0002-5130-8721

\section{REFERENCES}

1. Makri I, Papadima A, Lafioniati A, Pappas AB, George K, Nikolaos KE, et al. Sugammadex, a promising reversal drug. A review of clinical trials. Rev Recent Clin Trials 2011; 6: 250-5.

2. Debaene B, Plaud B, Dilly MP, Donati F. Residual paralysis in the PACU after a single intubating dose of nondepolarizing muscle relaxant with an intermediate duration of action. Anesthesiology 2003; 98: 1042-8.

3. Liu G, Wang R, Yan Y, Fan L, Xue J, Wang T. The efficacy and safety of sugammadex for reversing postoperative residual neuromuscular blockade in pediatric patients: a systematic review. Sci Rep 2017; 7: 5724.

4. Kara T, Ozbagriacik O, Turk HS, Isil CT, Gokuc O, Unsal O, et al. Sugammadex versus neostigmine in pediatric patients: a prospective randomized study. Rev Bras Anestesiol 2014; 64: 400-5.

5. Ammar AS, Mahmoud KM, Kasemy ZA. A comparison of sugammadex and neostigmine for reversal of rocuronium-induced neuromuscular blockade in children. Acta Anaesthesiol Scand 2017; 61:374-80.

6. Brull SJ, Kopman AF. Current status of neuromuscular reversal and monitoring: challenges and opportunities. Anesthesiology 2017; 126: 173-90.

7. Mayer M, Doenicke A, Hofmann A, Peter K. Onset and recovery of rocuronium (Org 9426) and vecuronium under enflurane anaesthesia. Br J Anaesth 1992; 69: 511-2.

8. Khuenl-Brady KS, Wattwil M, Vanacker BF, Lora-Tamayo JI, Rietbergen H, Alvarez-Gómez JA. Sugammadex provides faster reversal of vecuronium-induced neuromuscular blockade compared with neostigmine: a multicenter, randomized, controlled trial. Anesth Analg 2010; 110: 64-73.

9. Katz RL. Pyridostigmin (mestinon) as an antagonist of d-tubocurarine. Anesthesiology 1967; 28: 528-34.

10. Donati F, Lahoud J, McCready D, Bevan DR. Neostigmine, pyridostigmine and edrophonium as antagonists of deep pancuronium blockade. Can J Anaesth 1987; 34: 589-93.

11. Won YJ, Lim BG, Lee DK, Kim H, Kong MH, Lee IO. Sugammadex for reversal of rocuronium-induced neuromuscular blockade in pediatric patients: a systematic review and meta-analysis. Medicine (Baltimore) 2016; 95: e4678.

12. Ozgün C, Cakan T, Baltacı B, Başar H. Comparison of reversal and adverse effects of sugammadex and combination of - anticholinergic-anticholinesterase agents in pediatric patients. J Res Med Sci 2014; 19: 762-8.

13. Hendrickx JF, Eger EI 2nd, Sonner JM, Shafer SL. Is synergy the rule? A review of anesthetic interactions producing hypnosis and immobility. Anesth Analg 2008; 107: 494-506.

14. Lowry DW, Mirakhur RK, McCarthy GJ, Carroll MT, McCourt KC. Neuromuscular effects of rocuronium during sevoflurane, isoflurane, and intravenous anesthesia. Anesth Analg 1998; 87: 936-40.

15. Koyuncu O, Ozgur M, Akkurt C, Turhanoglu S, Akcora B, Celikkaya ME, et al. Induction with propofol decreases emergence agitation in pediatric patients. J Anesth Clin Res 2015; 6: 9. 